\title{
ARTICLE
}

Translational Therapeutics

\section{Systemic immune response induced by oxaliplatin-based neoadjuvant therapy favours survival without metastatic progression in high-risk rectal cancer}

\author{
Erta Kalanxhi ${ }^{1}$, Sebastian Meltzer ${ }^{1,2}$, Jakob Vasehus Schou ${ }^{3}$, Finn Ole Larsen ${ }^{3}$, Svein Dueland ${ }^{4}$, Kjersti Flatmark ${ }^{2,5,6}$, \\ Benny Vittrup Jensen ${ }^{3}$, Knut Håkon Hole ${ }^{7}$, Therese Seierstad ${ }^{7}$, Kathrine Røe Redalen ${ }^{1,8}$, Dorte Lisbet Nielsen ${ }^{3}$ and Anne Hansen Ree ${ }^{1,2}$
}

BACKGROUND: Systemic failure remains a challenge in rectal cancer. We investigated the possible systemic anti-tumour immune activity invoked within oxaliplatin-based neoadjuvant therapy.

METHODS: In two high-risk patient cohorts, we assessed the circulating levels of the fms-like tyrosine kinase 3 ligand (Flt3L), a factor reflecting both therapy-induced myelosuppression and activation of tumour antigen-presenting dendritic cells, at baseline and following induction chemotherapy and sequential chemoradiotherapy, both modalities containing oxaliplatin. The primary end point was progression-free survival (PFS).

RESULTS: In both cohorts, the median Flt3L level was significantly higher at completion of each sequential modality than at baseline. The 5-year PFS (most events being metastatic progression) was $68 \%$ and $71 \%$ in the two cohorts consisting of $33 \%$ and $52 \%$ T4 cases. In the principal cohort, a high Flt3L level following the induction chemotherapy was associated with low risk for a PFS event (HR: $0.15 ; P<0.01$ ). These patients also had available dose scheduling and toxicity data, revealing that oxaliplatin dose reduction during chemoradiotherapy, undertaken to maintain compliance to the radiotherapy protocol, was associated with advantageous PFS (HR: 0.47; $P=0.046)$.

CONCLUSION: In high-risk rectal cancer, oxaliplatin-containing neoadjuvant therapy may promote an immune response that favours survival without metastatic progression.

British Journal of Cancer (2018) 118:1322-1328; https://doi.org/10.1038/s41416-018-0085-y

\section{INTRODUCTION}

In locally advanced rectal cancer (LARC), locoregional neoadjuvant chemoradiotherapy (CRT) with a fluoropyrimidine in a noncytotoxic radiosensitising dose and resection of the residual tumour results in low local recurrence rates. ${ }^{1}$ However, metastatic progression remains a dominant cause of failure, as reported by $30-40 \%$ of cases in recent trials. ${ }^{2,3}$ Currently, no consensus exists to whether postoperative systemic therapy may reduce the metastatic risk in rectal cancer after neoadjuvant treatment. ${ }^{2,4,5}$ Alternative strategies involving neoadjuvant chemotherapy (NACT) prior to or immediately following the radiation have been investigated in a number of trials. ${ }^{6-13}$

In a phase II single-arm LARC study, patients were given oxaliplatin-/fluorouracil-containing induction NACT and sequential CRT with concomitant oxaliplatin and capecitabine. ${ }^{14}$ Acknowledging the concerns arising from protraction of the total treatment time, theoretically permitting tumour cell repopulation and selective pressure towards the survival of therapy-resistant cell clones, only 4 weeks of the induction NACT (two cycles of the Nordic FLOX regimen ${ }^{15}$ ) were given. This induction phase was found to be highly tolerable ${ }^{14}$ and alone led to substantial tumour volume reduction. ${ }^{16}$ Additionally, in a study population with a high proportion of T4 disease and lymph node involvement, 5-year progression-free survival (PFS; almost all of PFS events were metastatic progression) and overall survival (OS) were remarkably good. $^{14}$

Recent data from preclinical models ${ }^{17-20}$ and emerging clinical evidence ${ }^{21}$ indicate that tumour-targeting immune responses can be invoked by oxaliplatin-induced tumour cell death as shed tumour antigens, when captured and presented by dendritic cells, cause activation of cytotoxic T lymphocytes. In a similar fashion, radiation is not simply a cytotoxic agent within the treatment target volume, but may also exert systemic effects that are immune-mediated $^{22,23}$ and eradicate occult or clinically established tumour at distant sites (the so-called abscopal effect). ${ }^{24-26}$ When considering the clinical observations in our LARC study

\footnotetext{
${ }^{1}$ Department of Oncology, Akershus University Hospital, PO Box 1000, Lørenskog 1478, Norway; ${ }^{2}$ Institute of Clinical Medicine, University of Oslo, PO Box 1171, Oslo 0318,

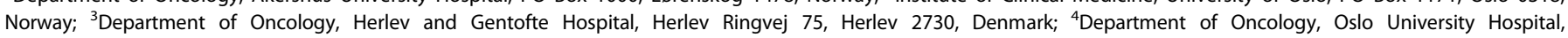

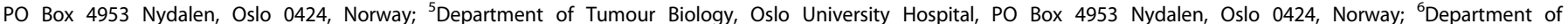

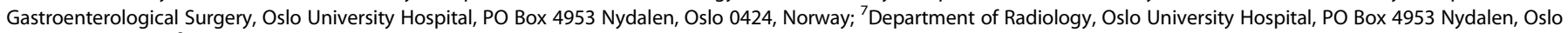
0424, Norway and ${ }^{8}$ Department of Physics, Norwegian University of Science and Technology, Høgskoleringen 5, Trondheim 7491, Norway

Correspondence: Anne Hansen. Ree (a.h.ree@medisin.uio.no)

These authors contributed equally: Erta Kalanxhi, Sebastian Meltzer.
} 
along with the possibility of oxaliplatin-induced immunogenic cell death, it was tempting to speculate that the short-course induction NACT might have unleashed the immune responses that would commission beneficial systemic effects in addition to the local clonogenic cell kill by the sequential CRT.

To investigate this hypothesis, we required a surrogate marker that would exhibit both the direct cytotoxic effects of chemotherapy and tumour-directed immunity. Hence, we analysed the circulating levels of the fms-related tyrosine kinase 3 ligand (Flt3L), a haematopoietic factor ${ }^{27}$ that reflects recovery from chemotherapy-induced myelosuppression ${ }^{28,29}$ and causes activation of mature dendritic cells. ${ }^{30-32}$ Specifically, Flt3L is a potent growth factor for a rare subset of professional antigen-presenting cells that stimulates cytotoxic T cells. ${ }^{33}$ Importantly, Flt3L has been shown to mediate abscopal effect of radiation in an experimental model, $^{31}$ and when administered to patients with metastatic colorectal cancer, it has led to expansion of the dendritic cell population both in the tumour periphery (locally) and the peripheral blood (systemically). ${ }^{34}$

In brief, we observed that a high serum Flt3L level following the two induction FLOX cycles was associated with low probability of a PFS event when oxaliplatin dose scheduling during the sequential CRT did not compromise the radiation delivery. Hence, an advantageous long-term outcome associated with maintenance of high circulating Flt3L during oxaliplatin-based neoadjuvant treatment in rectal cancer patients commonly prone to metastatic progression suggests that a beneficial systemic immune response may have been invoked.

\section{METHODS}

Patients

Patients eligible for the principal study, conducted in Oslo, Norway (NCT00278694), had histologically verified rectal adenocarcinoma that was either T4, T3 with mesorectal fascia margin of $3 \mathrm{~mm}$ or less, or a tumour of any $\mathrm{T}$ stage with lymph node involvement within $3 \mathrm{~mm}$ of this margin, as assessed by magnetic resonance imaging. ${ }^{14}$ With respect to eligibility, the only difference in the independent study cohort from Copenhagen, Denmark (NCT00964457), was that a margin of $\geq 1 \mathrm{~mm}$ of the mesorectal fascia excluded patients from participating in the study. ${ }^{35}$ Other eligibility criteria, evaluation procedures and follow-up have been described in detail previously. ${ }^{14,35}$ Anonymised biobank samples linked to de-identified selected patient data were transferred between institutions in accordance with the current regulations.

Treatment and end points of the Oslo cohort

This study was conducted to evaluate the safety, histologic tumour response and PFS of intensified neoadjuvant therapy. Induction NACT was given as two cycles of the Nordic FLOX regimen (oxaliplatin $85 \mathrm{mg} / \mathrm{m}^{2}$ on day 1 and bolus fluorouracil $500 \mathrm{mg} / \mathrm{m}^{2}$ and folinic acid $100 \mathrm{mg}$ on days 1 and 2 every second week). ${ }^{15}$ Sequential CRT consisted of radiation delivered five days per week to a total dose of $50 \mathrm{~Gy}$ in 25 fractions to the tumour bed and 48 Gy in 23 fractions to the regional lymph nodes, based on two-/ three-dimensional conformal planning models, with concomitant weekly oxaliplatin $50 \mathrm{mg} / \mathrm{m}^{2}$ and capecitabine $825 \mathrm{mg} / \mathrm{m}^{2}$ twice daily on days of radiotherapy. Treatment evaluation was done four weeks after the completion of preoperative therapy, and radical excision was planned 4-8 weeks after the evaluation. In accordance with national guidelines at that time, patients did not proceed to further therapy. Routine blood tests were done within the standard patient follow-up. The surrogate end points were histologic scorings of the resected tumour specimens (ypTN stage) and tumour regression grade (TRG), as presented previously. ${ }^{14,36-38}$ TRG1-2 and TRG3-5 were considered as good and poor tumour response, respectively. Magnetic resonance volumetry was undertaken after each sequential therapy, as described previously. ${ }^{39}$ Tumour volume changes from the baseline volume $\left(V_{\text {BASELINE }}\right)$ to after NACT $\left(\triangle V_{\text {NACT }}\right)$ and further from the volume after NACT to after CRT $\left(\Delta V_{\text {CRT }}\right)$ were calculated as $\Delta V_{\text {NACT }}=\left[\left(V_{\text {NACT }}-\right.\right.$ $\left.\left.V_{\text {BASELINE }}\right) / V_{\text {BASELINE }}\right] \times 100$ and $\Delta V_{\text {CRT }}=\left[\left(V_{\text {CRT }}-V_{\text {NACT }}\right) / V_{\text {NACT }}\right] \times 100$, respectively. The 73 patients included in this report were enrolled from the 5 October 2005 to the 3 March 2010. Censoring was done on the 8 August 2013, and PFS and death events were recorded a maximum of 5 years following pelvic surgery.

Treatment adjustments in the Oslo cohort

The neoadjuvant schedule was continuously adjusted according to toxicity, as assessed by the Common Terminology Criteria for Adverse Events (CTCAE) version 3.0, by reducing the doses of chemotherapy before radiation, and for myelosuppression grade 3 during CRT in particular, by reducing the dose of oxaliplatin more than that of capecitabine. Of further note in the context of the present report, oxaliplatin was reduced from CTCAE grade 2 neuropathy, while equal dose reductions of oxaliplatin and capecitabine were planned for CTCAE grade 2 myelosuppression and for CTCAE grade 2 and 3 diarrhoea (Supplementary Table S1). Formal recording of CTCAE scores was carried out at NACT and CRT completion.

Treatment and end points of the Copenhagen cohort Treatment of this cohort was not uniform. Patients were given NACT as 1-4 cycles (2-8 weeks) of oxaliplatin $85 \mathrm{mg} / \mathrm{m}^{2}$ every second week and capecitabine $650 \mathrm{mg} / \mathrm{m}^{2}$ twice a day continuously. Eighty-five percent of patients had radiation delivered five days per week to a total dose of $54 \mathrm{~Gy}$ in 27 fractions to the tumour bed and $48.6 \mathrm{~Gy}$ in 27 fractions to the regional lymph nodes, based on intensity-modulated planning models. For the remaining cases, alternative fractionation schedules or delivery techniques were used. All but one participant had concomitant capecitabine $825 \mathrm{mg} / \mathrm{m}^{2}$ twice daily continuously, but only $88 \%$ received weekly oxaliplatin $50 \mathrm{mg} / \mathrm{m}^{2}$ during radiotherapy. Finally, $57 \%$ of patients received $1-2$ cycles of preoperative chemotherapy (capecitabine with or without oxaliplatin) following CRT completion. ypTN stage and TRG were scored in the resected tumour specimens. Patients had pelvic surgery at a median of 5 (range, 2-19) weeks after CRT completion. PFS and OS status was censored at a median of 64 (range, 2-67) months after study enrolment; i.e., at the available time point closest to 5 years after the pelvic surgery for each patient.

Measurement of serum Flt3L levels

Patients had serum sampled at baseline and following the completion of NACT (post NACT) and CRT (post CRT). Additionally, the Oslo study patients had serum collected at treatment evaluation prior to surgery. The collected samples were stored at $-80^{\circ} \mathrm{C}$ until analysis. In samples from the Oslo cohort, serum Flt3L was analysed with the Human Flt-3 Ligand Quantikine ${ }^{\circledR}$ ELISA Kit (R\&D Systems). For practical reasons, analysis of the Copenhagen samples was performed with a customised Luminex Multiplex Assay (R\&D Systems). One-to-two dilution and two technical replicates were done for all samples. In 39 randomly chosen samples covering all three sampling points from a total of 19 patients of the Copenhagen cohort, strong correlation $(r=0.96$; $P<0.01$; by Pearson product correlation) was found between Flt3L levels measured by the single-parameter and multiplex assays (Supplementary Fig. S1).

\section{Statistical analysis}

Analyses were performed using IBM SPSS Statistics for Mac version 24.0 and GraphPad Prism version 6.0h. Groups were compared either using unpaired one-way analysis of variance with Bonferroni's test for multiple comparisons or Student's $t$-test, as appropriate. Correlations were determined by Pearson product correlation analysis after transformation of the data to natural 
logarithms. PFS was calculated from the time of study enrolment to the date of recurrent disease (diagnosis of local recurrence or distant metastasis), death of any cause, or end of follow-up,

\begin{tabular}{|c|c|c|}
\hline & $\begin{array}{l}\text { Oslo } \\
N(\%)\end{array}$ & $\begin{array}{l}\text { Copenhagen } \\
N(\%)\end{array}$ \\
\hline Total cohort & $73(100)$ & $65(100)$ \\
\hline \multicolumn{3}{|l|}{ Age, years } \\
\hline Median (range) & $59(30-73)$ & $67(41-78)$ \\
\hline \multicolumn{3}{|l|}{ Gender } \\
\hline Male & $42(58)$ & $39(60)$ \\
\hline Female & $31(42)$ & $26(40)$ \\
\hline \multicolumn{3}{|l|}{ T stage } \\
\hline $\mathrm{T} 2$ & $5(7)$ & 0 \\
\hline T3 & $44(60)$ & $31(48)$ \\
\hline $\mathrm{T} 4$ & $24(33)$ & $34(52)$ \\
\hline \multicolumn{3}{|l|}{$\mathrm{N}$ stage } \\
\hline No & $9(12)$ & $16(25)$ \\
\hline $\mathrm{N} 1$ & $8(11)$ & $15(23)$ \\
\hline N2 & $55(75)$ & $21(32)$ \\
\hline ND & $1(1.4)$ & $13(20)$ \\
\hline \multicolumn{3}{|l|}{ ypT stage } \\
\hline урто & $15(21)$ & $7(11)$ \\
\hline урT1 & $7(10)$ & $3(4.6)$ \\
\hline урт2 & $17(23)$ & $9(14)$ \\
\hline урт3 & $22(30)$ & $30(46)$ \\
\hline урT4 & $11(15)$ & $11(17)$ \\
\hline ND & $1(1.4)$ & $5(7.7)$ \\
\hline \multicolumn{3}{|l|}{ ypN stage } \\
\hline ypNo & $52(71)$ & $47(72)$ \\
\hline ypN1 & $16(22)$ & $10(15)$ \\
\hline ypN2 & $4(5)$ & $3(5)$ \\
\hline ND & $1(1.4)$ & $5(7.7)$ \\
\hline \multicolumn{3}{|l|}{ TRG score } \\
\hline TRG1-2 & $53(73)$ & $23(35)$ \\
\hline TRG3-5 & $19(26)$ & $35(54)$ \\
\hline ND & $1(1.4)$ & $7(11)$ \\
\hline
\end{tabular}

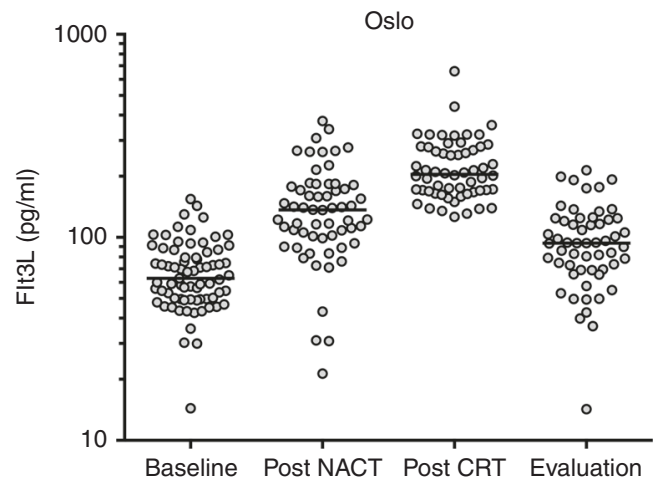

whichever occurred first. Associations between the selected variables and the PFS were modelled with univariate Cox regression analysis and further analysed by multivariate Cox regression using backwards stepwise conditional selection, as appropriate, and the results were expressed as hazard ratio (HR) with $95 \%$ confidence interval $(\mathrm{Cl})$. All reported $P$ values were for two-sided tests with significance level set less than 0.05 .

\section{RESULTS}

Patient characteristics and serum Flt3L levels-Oslo cohort The 73 patients mainly presented with T3 $(60 \%)$ or T4 (33\%) disease (Table 1). Median age was 59 years. As seen in Fig. 1, the median serum Flt3L level increased from 62.9 (range, 14.4-154) $\mathrm{pg} / \mathrm{ml}(N=73)$ at baseline to 137 (range, 21.3-374) $\mathrm{pg} / \mathrm{ml}(N=60)$ post NACT and further to 205 (range, 126-658) pg/ml $(N=58)$ post CRT. At evaluation, the median Flt3L had fallen to 93.7 (range, 14.2-214) $\mathrm{pg} / \mathrm{ml}(N=56)$. The serum Flt3L measures (mean \pm SD) during the neoadjuvant treatment course, including that at evaluation 4 weeks after completion of the neoadjuvant therapy, were significantly different from baseline $(P<0.0001$; by unpaired one-way analysis of variance with Bonferroni's test for multiple comparisons).

Cytotoxicity over the course of neoadjuvant therapy As both post NACT and post CRT serum sampling points immediately followed the completion of the preceding sequential therapy, we could investigate whether the individual patient's serum Flt3L alteration at each of the sampling points (fold-change from the preceding one) might correlate with myelosuppressive effects (in terms of fold-change of absolute peripheral blood

Table 2. Treatment-induced changes in circulating Flt3L levels and correlations with cytotoxic effects - Oslo study

\begin{tabular}{|c|c|c|c|c|c|c|}
\hline \multirow[t]{2}{*}{ Measure of cytotoxicity } & \multicolumn{3}{|c|}{ Post NACT } & \multicolumn{3}{|c|}{ Post CRT } \\
\hline & $N$ & $r$ & $P$ & $N$ & $r$ & $P$ \\
\hline Haemoglobin & 56 & -0.38 & $<0.01$ & 49 & 0.02 & 0.87 \\
\hline Thrombocytes & 56 & -0.07 & 0.62 & 49 & -0.34 & 0.02 \\
\hline Neutrophils & 56 & -0.36 & 0.01 & 49 & -0.19 & 0.19 \\
\hline Lymphocytes & 54 & 0.27 & 0.05 & 47 & 0.11 & 0.46 \\
\hline Monocytes & 53 & 0.07 & 0.60 & 40 & 0.47 & 0.01 \\
\hline Tumour $\Delta V^{a}$ & 49 & -0.32 & 0.03 & 43 & 0.07 & 0.67 \\
\hline
\end{tabular}

CRT chemoradiotherapy, Flt3L fms-like tyrosine kinase 3 ligand, NACT neoadjuvant chemotherapy ${ }^{\mathrm{a}} \Delta V$ denotes $\Delta V_{\mathrm{NACT}}$ post NACT and $\Delta V_{\mathrm{CRT}}$ post CRT

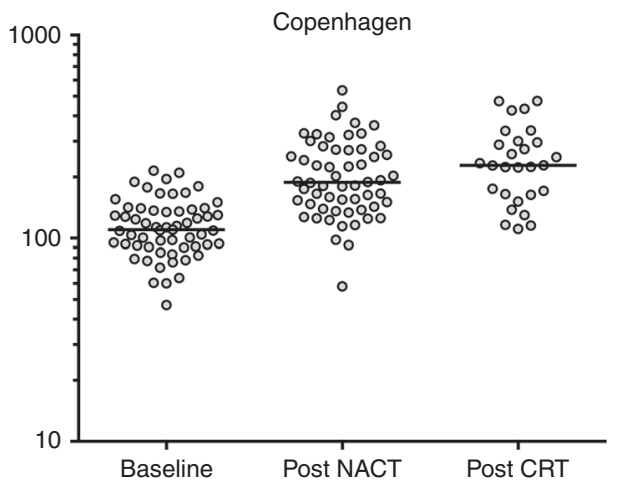

Fig. 1 Serum Flt3L levels during the neoadjuvant treatment course in the Oslo and Copenhagen patient cohorts. The horizontal line in each data cluster represents the median value. CRT chemoradiotherapy, Flt3L fms-like tyrosine kinase 3 ligand, NACT neoadjuvant chemotherapy 
Table 3. Circulating Flt3L levels during the treatment course and PFSOslo study

\begin{tabular}{llll}
\hline Sampling point & $N$ & $\mathrm{HR}(95 \% \mathrm{Cl})^{\mathrm{a}}$ & $P$ \\
\hline Baseline & 73 & $9.5(1.2-80)$ & 0.04 \\
Post NACT & 60 & $0.15(0.04-0.55)$ & $<0.01$ \\
Post CRT & 58 & $1.1(0.17-7.7)$ & 0.90 \\
Evaluation & 56 & $0.43(0.15-1.2)$ & 0.11 \\
\hline
\end{tabular}

Cl confidence interval, CRT chemoradiotherapy, Flt3L fms-like tyrosine kinase 3 ligand, HR hazard ratio, NACT neoadjuvant chemotherapy, PFS progression-free survival ${ }^{\text {a }}$ Low HR indicates favourable PFS (low probability of a PFS event) with high Flt3L level

measures) and the immediate tumour response $(\Delta V)$ to the respective treatment, as a manifestation of its cytotoxicity (Table 2). At NACT completion, a significant negative correlation was found between changes in levels of Flt3L and those of haemoglobin and neutrophils. The corresponding correlation with thrombocytes at CRT completion reflected a more protracted effect, commonly observed with oxaliplatin-containing regimens. Interestingly, at this time point there was also a positive correlation between changes in the levels of Flt3L and monocytes. Of further note, the immediate treatment response in terms of relative tumour volume shrinkage (negative $\Delta V$ value) was correlated with an increase in the Flt3L level only after induction NACT. In this regard, the histologic tumour response to radiation in rectal cancer is not manifest until 6-8 weeks following administration of the total dose. ${ }^{40}$

Serum Flt3L levels and treatment outcome

In further support of a potential connection between myelosuppressive effects and beneficial treatment response, absolute serum Flt3L levels at baseline and post NACT were found to be significantly higher in patients with good histologic tumour response (TRG1-2), as compared to those with poor response (TRG3-5) following the neoadjuvant therapy; however, no difference was seen with regard to histologic ypTN stage (Supplementary Table S2). When censored, the median follow-up time was 65 (range, 3-66) months. The majority of PFS events consisted of metastatic progression, all of which occurred after completion of the neoadjuvant therapy. The 5 -year PFS was $68 \%$. As shown in Table 3, post NACT serum Flt3L level was strongly associated with the outcome; the higher circulating Flt3L level following the shortcourse FLOX therapy, the better PFS (HR: 0.15 ; 95\% Cl: $0.04-0.55$; $P<0.01$ for a PFS event with high Flt3L level; by multivariate Cox regression analysis). This feature applied specifically to T4 cases (Supplementary Table S3). Pertaining to the entire Oslo cohort, post NACT Flt3L level was statistically stronger as a prognostic marker than baseline $T$ stage, but not significant when compared to postoperative ypT stage (Supplementary Table S4); histologic tumour response is regarded as a superior determinant of recurrence risk after neoadjuvant CRT. ${ }^{1}$ Moreover, a long interval between disease progression and death was reflected by a 5 -year OS of $88 \%$. Correspondingly, for cases with recorded PFS events or death at censoring, median time to progression was 14 (range, 3-52) months, but 31 (range, 8-66) months for death.

Adjustment of oxaliplatin dose and PFS

As shown in Table 4, the induction NACT was well tolerated and $90 \%$ of patients received the intended dose. However, the majority of patients experienced chemotherapy dose reduction during the sequential CRT, consistent with dose adjustments of both chemotherapeutics planned already at CTCAE grade 2 myelosuppression or diarrhoea (Supplementary Table S1). Only $19 \%$ of patients received the full-prescribed chemotherapy doses
Table 4. Chemotherapy dose adjustments during the treatment course and PFS-Oslo study

\begin{tabular}{lllll}
\hline Therapy & Dose & $N(\%)$ & HR $(95 \%$ Cl) & $P$ \\
\hline Induction NACT & Full & $66(90)$ & & \\
& Reduced & $7(10)$ & $0.64(0.15-2.7)$ & 0.54 \\
$\begin{array}{l}\text { Sequential CRT- } \\
\text { oxaliplatin }\end{array}$ & Full & $17(23)$ & & \\
& Reduced & $56(77)$ & $0.47(0.22-0.99)$ & 0.046 \\
$\begin{array}{l}\text { Sequential CRT- } \\
\text { capecitabine }\end{array}$ & Full & $24(33)$ & & \\
& Reduced & $49(67)$ & $2.8(0.75-10)$ & 0.13 \\
$\begin{array}{l}\text { Entire neoadjuvant } \\
\text { treatment }\end{array}$ & Full & $14(19)$ & & \\
& Reduced & $59(81)$ & NA & NA \\
\hline $\begin{array}{l}\text { Cl confidence interval, CRT chemoradiotherapy, HR hazard ratio, NA not } \\
\text { applicable, NACT neoadjuvant chemotherapy, PFS progression-free survival } \\
\text { a Low HR indicates favourable PFS (low probability of a PFS event) with } \\
\text { chemotherapy dose reduction }\end{array}$ & & & \\
\hline
\end{tabular}

over the entire neoadjuvant treatment; while $67 \%$ had reduction in capecitabine during CRT, $77 \%$ did not receive the full-oxaliplatin dose (Table 4). Per protocol, cases with grade 3 neutropenia would have oxaliplatin dose lowered more than capecitabine dose, and cases with grade $\geq 2$ neuropathy would have oxaliplatin administration interrupted or discontinued (Supplementary Table S1). Over the active neoadjuvant treatment, a total of 42 CTCAE grade 2-3 events of neutropenia, diarrhoea, paraesthesia, or hand-foot syndrome were reported (Supplementary Table S5). During CRT (Table 4), 29 patients (40\% of the 73 study participants) had reduction in oxaliplatin dose because of neutropenia, diarrhoea and paraesthesia (32 events in total; Supplementary Table S5), leaving 27 patients (37\% of participants) with oxaliplatin dose adjustment of other reasons such as fatigue, fever, vomiting and anorexia. The details on all recorded CTCAE grade 3-5 adverse events have been reported previously. ${ }^{14}$

As further shown in Table 4, reduction in the planned oxaliplatin dose during CRT was by a narrow margin associated with advantageous PFS (HR: 0.47; 95\% Cl: $0.22-0.99 ; P=0.046$ for a PFS event in patients with dose reduction; by multivariate Cox regression analysis). However, this association was lost when the time to reduction in the neoadjuvant course was included as a covariate in the analysis (data not shown). This analysis was done because PFS was calculated from the time of study enrolment and therefore, dose adjustment at later time points might have led to bias in the analysis. Moreover, in CRT, capecitabine is used for its radiosensitising properties at a non-cytotoxic dose, ${ }^{41}$ and reduced doses were not associated with PFS (Table 4). With respect to the radiotherapy, only two of the 73 participants $(2.7 \%)$ did not receive the total dose of $\geq 50 \mathrm{~Gy}$; five of the seven patients who had interruption in radiation delivery (owing to CTCAE grade 3 diarrhoea before CRT completion; Supplementary Table S1) received compensation to ensure biological equivalence in efficacy. ${ }^{42}$ Hence, for a majority of patients, the reported side effects of chemotherapy did not compromise delivery of the fullprescribed radiation dose.

Serum Flt3L levels-Copenhagen cohort

In this cohort, which met the criterion of comprising LARC patients given sequential neoadjuvant therapy along with simultaneous serial serum sampling and available long-term outcome data, 20\% and $80 \%$ of patients had received 2-4 weeks or 6-8 weeks, respectively, of oxaliplatin-based induction NACT. A total of 65 patients were older (median age 67 years) with a higher 
1326

percentage of T4 cases (52\%), when compared to the Oslo cohort (Table 1). With regard to serum Flt3L levels, as assessed with an assay that gave Flt3L measures at higher concentrations (Supplementary Fig. S1), a similar pattern of changes was observed during the treatment course (Fig. 1). The median Flt3L level was 110 (range, 47.0-215) $\mathrm{pg} / \mathrm{ml}(N=59)$ at baseline that increased significantly to 188 (range, 58.1-535) $\mathrm{pg} / \mathrm{ml}(N=58)$ post NACT and 228 (range, $111-474) \mathrm{pg} / \mathrm{ml}(N=28)$ post CRT. Of note, the relative increment in circulating Flt3L from baseline to NACT completion was higher in the Oslo cohort $(P=0.03$ on comparison). Further on, compared to the Oslo counterparts, the Copenhagen patients had considerably worse histologic tumour response (ypT3-4 stage and TRG3-5 score; Table 1), which might be related to the greater number of T4 cases. Other dissimilarities of note were the 5-year PFS and OS that were strikingly equal (71\% and 69\%, respectively, for the entire cohort), as were also the median times to progression [22 (range, 9-63) months] and death [29 (range, 2-65) months] for cases with these events at censoring. Finally, no association was found between PFS and serum Flt3L levels at any sampling point (Supplementary Table S3).

\section{DISCUSSION}

Circulating levels of the haematopoietic factor Flt3L increased in two independent cohorts of LARC patients during induction NACT and further over sequential CRT, both modalities containing oxaliplatin. In the Oslo cohort, the higher Flt3L level following the induction NACT, the more favourable 5-year PFS without metastatic progression. This cohort had available dose scheduling and toxicity data, which revealed that oxaliplatin dose reduction during CRT, undertaken to avert breach of radiation delivery, was associated with the advantageous PFS. Elevation of the circulating Flt3L level reflects recovery from chemotherapy-induced myelosuppression, ${ }^{28,29}$ and it expands the pool of professional antigen-presenting dendritic cells. ${ }^{33}$ Despite missing validation in the Copenhagen cohort, the present data support the notion that oxaliplatin-based neoadjuvant therapy, when administered at an intensity sufficient to maintain patient compliance to the radiotherapy protocol, may promote an immune response that enables eradication of occult microscopic tumour at distant sites (abscopal effect) in LARC patients prone to develop metastatic disease.

Metastatic progression in spite of local tumour control represents a major challenge in the treatment of LARC. Hence, varying combinations of multimodal therapy have been evaluated with the aim of preventing systemic dissemination. Chemotherapy consisting of oxaliplatin and a fluoropyrimidine has been administered before CRT (in trials not randomised for the induction therapy) or followed short-course radiation prior to surgery (the experimental arm of the recently concluded RAPIDO trial; efficacy data are not yet reported) in an attempt to deliver adequate systemic therapy for metastasis prevention without compromising local disease control. ${ }^{6-13}$ Because radiation has the ability to deliver cytotoxic effects in a focused tumour volume, it has been argued that improved systemic outcome may be achieved by intensifying the local effects that enhance elimination of clonogenic cells. ${ }^{43}$ In that regard, only two of seven randomised studies that have evaluated the potentially radiosensitising effect of concomitant oxaliplatin in fluoropyrimidine-based CRT met the primary efficacy end point, although in several of the studies fewer experimental arm patients seemed to have metastatic disease at surgery. ${ }^{3,44-49}$ The same two studies showed high patient compliance to the chosen dose scheduling of oxaliplatin; ${ }^{3,45}$ c.f., Supplementary Table S6. In the present study cohorts, induction oxaliplatin-based NACT was administered before long-course CRT where oxaliplatin was given concomitantly for the majority of patients, with the intention of intensifying local radiation effects but with minimal protraction of the total neoadjuvant treatment time and maintenance of compliance. This approach might have caused repetitive myelosuppression (because oxaliplatin was dosed at sufficient intensity for the individual patient), resulting in elevated circulating Flt3L levels as a rescue response during the neoadjuvant treatment course, and thereby enhanced the pool of functionally active immune cells and the mediation of systemic tumour-targeting effects. Several observations supported this notion.

Firstly, serum Flt3L levels increased as an immediate response to induction NACT and were higher than baseline even 4 weeks after completion of the full neoadjuvant therapy. Secondly, the initial relative increase in Flt3L levels was correlated with the relative decline in neutrophils, which has also been observed previously. ${ }^{28}$ Additionally, at CRT completion, Flt3L induction was correlated with an increase in the monocyte count, an indirect measure of the presumed haematopoietic effect on dendritic cells appearing over the treatment course. Moreover, in the Oslo cohort, the increase in serum Flt3L levels during the induction NACT was correlated with tumour volume shrinkage (local response), and a high absolute Flt3L level at this instance was associated with a good TRG score (local response) and favourable 5-year PFS (systemic response), where the local response measures are surrogate end points for the final outcome. Of further note, median OS and 5-year OS were considerably better than the corresponding PFS measures. Finally, the observed association between oxaliplatin dose reduction during CRT and an advantageous long-term outcome supported the notion of maintenance of a systemic anti-tumour immune response, ${ }^{50}$ strengthened by the finding of serum Flt3L elevation during induction NACT and further during sequential CRT in the independent Copenhagen cohort.

However, in the Oslo cohort, patients' post CRT Flt3L level (significantly different from baseline) was not predictive of PFS. Although the observed association between post NACT Flt3L level and PFS may be a chance finding, it would not be entirely surprising that the timing of the Flt $3 \mathrm{~L}$ increase may be important. Remaining speculative without further studies, it could still be that the two cycles of the Nordic FLOX regimen unleashed the advantageous systemic responses just prior to the administration of the main therapeutic modality (CRT). In this context, it is worth noting that the heterogeneous neoadjuvant regimens in the Copenhagen cohort might have contributed to the lack of association between PFS and circulating Flt3L.

There are several weaknesses inherent to the present study. Importantly, this set of analyses was not planned at the time of trial conduct, but prompted by the evidence that appeared as we started to look at clinical outcome data. Hence, none of the performed analyses had been prespecified in a statistical analysis plan. Both study cohorts are relatively small, but our attempts in identifying other independent studies holding all of the required criteria have been unsuccessful. There were considerable dissimilarities between the two cohorts. The Oslo and Copenhagen patients had different distribution of age and TN stage, and they did not receive exactly the same neoadjuvant treatment. The former cohort had 4 weeks of oxaliplatincontaining NACT, however, $80 \%$ of the latter cohort had this treatment for a longer period (6-8 weeks). The relative increment in the circulating Flt3L during NACT was significantly lower in the latter and not associated with PFS, which might indicate that oxaliplatin had not been dosed at sufficient intensity. More than $10 \%$ of Copenhagen patients did not receive concomitant oxaliplatin (to maintain the presumed optimum Flt3L level for an advantageous long-term outcome), and the median and 5-year PFS and OS measures were strikingly similar in this cohort. Despite the emerging data demonstrating that oxaliplatin is responsible for 'on-target' immunogenic cell death ${ }^{17-21}$ and 'off-target' anti-tumour immune responses, ${ }^{21}$ this drug was one component with other cytotoxic therapies in the 
multimodal treatment regimens. It is also important to keep in mind that the radiotherapy technique used in the Oslo cohort was based on two-/three-dimensional conformal planning that might have caused irradiation of pelvic bone marrow, thus contributing to the observed haematological toxicity. A relevant dose-volume parameter to assess such contribution has not been determined. Furthermore, we found that oxaliplatin dose adjustment as such, and not the grade of severity of myelosuppression, ${ }^{51}$ was prognostic for improved survival. The association was not strong and was lost when the time to dose adjustment in the neoadjuvant course was included as a covariate in the analysis. However, the analysis was done for a preset accumulative dose of chemotherapy in a definite neoadjuvant regimen. Hence, the data indicate that oxaliplatin dose reduction as such, and not the exact time for this event within the neoadjuvant course, was relevant for patient survival. Finally, data on prophylactic use of granulocyte colonystimulating factor were not recorded within the study.

In conclusion, oxaliplatin-containing neoadjuvant therapy that causes normal tissue toxicity that is sufficient to maintain a high level of circulating Flt3L but does not compromise patient compliance to the radiotherapy, may favour survival without metastatic progression of LARC patients otherwise prone to this event. In the era of identifying the optimum context of immune-modulating therapies, one recommendation might use a short-course oxaliplatin-based therapy to condition the immune system and transform LARC, or metastatic colorectal cancer, into an immunogenic disease before sequential immune checkpoint blockade, currently in the forefront of combination cancer therapies.

\section{ACKNOWLEDGEMENTS}

We thank Professor Julia Sidenius Johansen for managing the study biobank at Department of Oncology, Herlev and Gentofte Hospital and Mrs. Marianne Johansen for her excellent work as study nurse in the Oslo study. South-Eastern Norway Regional Health Authority (grant 2013002 to K.R.R. and grants 2013101 and 2014010 to A.H.R.), Akershus University Hospital (grants 2015017 and 2016024 to A.H.R.) and the Norwegian Cancer Society (grant 6803027 to A.H.R.).

\section{AUTHOR CONTRIBUTIONS}

E.K., S.M. and A.H.R. contributed to the concept and design of the study. J.V.S., F.O.L. S.D., K.F., B.V.J., D.L.N. and A.H.R. managed the patients and databases and provision of clinical information. K.H.H., T.S. and K.R.R. managed the imaging database and tumour volumetry. E.K. and S.M. performed all analyses. E.K., S.M. and A.H.R. drafted the manuscript. All authors read and approved the final version.

\section{ADDITIONAL INFORMATION}

Supplementary information is available for this paper at https://doi.org/10.1038/ s41416-018-0085-y.

Competing interests: The authors declare no competing interests.

Ethics approval and consent to participate: The study protocols were approved by the Regional Committee for Medical and Health Research Ethics of South Norway (REK-S-05059) and Committees for Research Ethics of the Capital Region of Denmark (H-C-2008-0001 and H-A-2009-019). The studies were performed in accordance with the Declaration of Helsinki. Written informed consent was required for participation.

Note: This work is published under the standard license to publish agreement. After 12 months the work will become freely available and the license terms will switch to a Creative Commons Attribution 4.0 International licence (CC BY 4.0).

\section{REFERENCES}

1. Aklilu, M. \& Eng, C. The current landscape of locally advanced rectal cancer. Nat. Rev. Clin. Oncol. 8, 649-659 (2011).
2. Bosset, J. F. et al. Fluorouracil-based adjuvant chemotherapy after preoperative chemoradiotherapy in rectal cancer: long-term results of the EORTC 22921 randomised study. Lancet Oncol. 15, 184-190 (2014).

3. Rödel, C. et al. Oxaliplatin added to fluorouracil-based preoperative chemoradiotherapy and postoperative chemotherapy of locally advanced rectal cancer (the German CAO/ARO/AIO-04 study): final results of the multicentre, open-label, randomised, phase 3 trial. Lancet Oncol. 16, 979-989 (2015).

4. Petersen S. H., Harling H., Kirkeby L. T., Wille-Jørgensen P., \& Mocellin S. Postoperative adjuvant chemotherapy in rectal cancer operated for cure. Cochrane Database Syst Rev 2012; CD004078.

5. Breugom, A. J. et al. Adjuvant chemotherapy after preoperative (chemo)radiotherapy and surgery for patients with rectal cancer: a systematic review and meta-analysis of individual patient data. Lancet Oncol. 16, 200-207 (2015).

6. Calvo, F. A. et al. Improved incidence of pT0 downstaged surgical specimens in locally advanced rectal cancer (LARC) treated with induction oxaliplatin plus 5fluorouracil and preoperative chemoradiation. Ann. Oncol. 17, 1103-1110 (2006).

7. Chau, l. et al. Neoadjuvant capecitabine and oxaliplatin followed by synchronous chemoradiation and total mesorectal excision in magnetic resonance imagingdefined poor-risk rectal cancer. J. Clin. Oncol. 24, 668-674 (2006).

8. Koeberle, D. et al. Phase II study of capecitabine and oxaliplatin given prior to and concurrently with preoperative pelvic radiotherapy in patients with locally advanced rectal cancer. Br. J. Cancer 98, 1204-1209 (2008).

9. Gunnlaugsson, A. et al. Multicentre phase II trial of capecitabine and oxaliplatin in combination with radiotherapy for unresectable colorectal cancer: the CORGI-L Study. Eur. J. Cancer 45, 807-813 (2009).

10. Chua, Y. J. et al. Neoadjuvant capecitabine and oxaliplatin before chemoradiotherapy and total mesorectal excision in MRI-defined poor-risk rectal cancer: a phase 2 trial. Lancet Oncol. 11, 241-248 (2010).

11. Dewdney, A. et al. Multicenter randomized phase II clinical trial comparing neoadjuvant oxaliplatin, capecitabine, and preoperative radiotherapy with or without cetuximab followed by total mesorectal excision in patients with highrisk rectal cancer (EXPERT-C). J. Clin. Oncol. 30, 1620-1627 (2012).

12. Schou, J. V. et al. Induction chemotherapy with capecitabine and oxaliplatin followed by chemoradiotherapy before total mesorectal excision in patients with locally advanced rectal cancer. Ann. Oncol. 23, 2627-2633 (2012).

13. Nilsson, P. J. et al. Short-course radiotherapy followed by neo-adjuvant chemotherapy in locally advanced rectal cancer--the RAPIDO trial. Bmc. Cancer 13, 279 (2013)

14. Dueland, S. et al. Oxaliplatin-containing preoperative therapy in locally advanced rectal cancer: local response, toxicity and long-term outcome. Clin. Oncol. (R. Coll. Radiol.). 28, 532-539 (2016).

15. Tveit, K. M. et al. Phase III trial of cetuximab with continuous or intermittent fluorouracil, leucovorin, and oxaliplatin (Nordic FLOX) versus FLOX alone in firstline treatment of metastatic colorectal cancer: the NORDIC-VII study. J. Clin. Oncol. 30, 1755-1762 (2012).

16. Flatmark, K. et al. Individual tumor volume responses to short-course oxaliplatincontaining induction chemotherapy in locally advanced rectal cancer - targeting the tumor for radiation sensitivity? Radiother. Oncol. 119, 505-511 (2016).

17. Tesniere, A. et al. Immunogenic death of colon cancer cells treated with oxaliplatin. Oncogene 29, 482-491 (2010).

18. Zitvogel, L. et al. Immunogenic tumor cell death for optimal anticancer therapy: the calreticulin exposure pathway. Clin. Cancer Res. 16, 3100-3104 (2010).

19. Gou, H. F., Huang, J., Shi, H. S., Chen, X. C. \& Wang, Y. S. Chemo-immunotherapy with oxaliplatin and interleukin-7 inhibits colon cancer metastasis in mice. PLOS ONE 9, e85789 (2014).

20. Pfirschke, C. et al. Immunogenic chemotherapy sensitizes tumors to checkpoint blockade therapy. Immunity 44, 343-354 (2016).

21. Galluzzi, L., Buque, A., Kepp, O., Zitvogel, L. \& Kroemer, G. Immunological effects of conventional chemotherapy and targeted anticancer agents. Cancer Cell. 28, 690-714 (2015).

22. Golden, E. B. \& Apetoh, L. Radiotherapy and immunogenic cell death. Semin. Radiat. Oncol. 25, 11-17 (2015).

23. Demaria, S., Pilones, K. A., Vanpouille-Box, C., Golden, E. B. \& Formenti, S. C. The optimal partnership of radiation and immunotherapy: from preclinical studies to clinical translation. Radiat. Res. 182, 170-181 (2014).

24. Formenti, S. C. \& Demaria, S. Systemic effects of local radiotherapy. Lancet Oncol. 10, 718-726 (2009).

25. Postow, M. A. et al. Immunologic correlates of the abscopal effect in a patient with melanoma. N. Engl. J. Med. 366, 925-931 (2012).

26. Whiteside, T. L., Demaria, S., Rodriguez-Ruiz, M. E., Zarour, H. M. \& Melero, I. Emerging opportunities and challenges in cancer immunotherapy. Clin. Cancer Res. 22, 1845-1855 (2016).

27. Antonysamy, M. A. \& Thomson, A. W. Flt3 ligand ( $F L)$ and its influence on immune reactivity. Cytokine 12, 87-100 (2000). 
28. Greystoke, A. et al. Assessment of circulating biomarkers for potential pharmacodynamic utility in patients with lymphoma. Br. J. Cancer 104, 719-725 (2011).

29. Wodnar-Filipowicz, A. et al. Flt3 ligand level reflects hematopoietic progenitor cell function in aplastic anemia and chemotherapy-induced bone marrow aplasia. Blood 88, 4493-4499 (1996).

30. Maraskovsky, E. et al. Dramatic increase in the numbers of functionally mature dendritic cells in Flt3 ligand-treated mice: multiple dendritic cell subpopulations identified. J. Exp. Med. 184, 1953-1962 (1996).

31. Demaria, S. et al. lonizing radiation inhibition of distant untreated tumors (abscopal effect) is immune mediated. Int. J. Radiat. Oncol. Biol. Phys. 58, 862-870 (2004).

32. Merad, M., Sathe, P., Helft, J., Miller, J. \& Mortha, A. The dendritic cell lineage: ontogeny and function of dendritic cells and their subsets in the steady state and the inflamed setting. Annu. Rev. Immunol. 31, 563-604 (2013).

33. Broz, M. L. et al. Dissecting the tumor myeloid compartment reveals rare activating antigen-presenting cells critical for T cell immunity. Cancer Cell 26, 638-652 (2014).

34. Morse, M. A. et al. Preoperative mobilization of circulating dendritic cells by Flt3 ligand administration to patients with metastatic colon cancer. J. Clin. Oncol. 18, 3883-3893 (2000).

35. Larsen, F. O. et al. Capecitabine and oxaliplatin before, during, and after radiotherapy for high-risk rectal cancer. Clin. Colorectal Cancer 16, e7-e14 (2017).

36. Hektoen, H. H. et al. Early increase in circulating carbonic anhydrase IX during neoadjuvant treatment predicts favourable outcome in locally advanced rectal cancer. BMC Cancer 15, 543 (2015)

37. Meltzer, S. et al. Systemic release of osteoprotegerin during oxaliplatincontaining induction chemotherapy and favorable systemic outcome of sequential radiotherapy in rectal cancer. Oncotarget 7, 34907-34917 (2016).

38. Kalanxhi, E. et al. Circulating proteins in response to combined-modality therapy in rectal cancer identified by antibody array screening. BMC Cancer 16, 536 (2016).

39. Seierstad, T. et al. MRI volumetry for prediction of tumour response to neoadjuvant chemotherapy followed by chemoradiotherapy in locally advanced rectal cancer. Br. J. Radiol. 88, 20150097 (2015).

40. Wolthuis, A. M. et al. Impact of interval between neoadjuvant chemoradiotherapy and TME for locally advanced rectal cancer on pathologic response and oncologic outcome. Ann. Surg. Oncol. 19, 2833-2841 (2012).
41. Seiwert, T. Y., Salama, J. K. \& Vokes, E. E. The concurrent chemoradiation paradigm--general principles. Nat. Clin. Pract. Oncol. 4, 86-100 (2007).

42. Bese, N. S., Hendry, J. \& Jeremic, B. Effects of prolongation of overall treatment time due to unplanned interruptions during radiotherapy of different tumor sites and practical methods for compensation. Int. J. Radiat. Oncol. Biol. Phys. 68, 654-661 (2007)

43. Lawrence, T. S., Haffty, B. G. \& Harris, J. R. Milestones in the use of combinedmodality radiation therapy and chemotherapy. J. Clin. Oncol. 32, 1173-1179 (2014).

44. Aschele, C. et al. Primary tumor response to preoperative chemoradiation with or without oxaliplatin in locally advanced rectal cancer: pathologic results of the STAR-01 randomized phase III trial. J. Clin. Oncol. 29, 2773-2780 (2011).

45. Deng, Y. et al. Modified FOLFOX6 with or without radiation versus fluorouracil and leucovorin with radiation in neoadjuvant treatment of locally advanced rectal cancer: initial results of the Chinese FOWARC multicenter, open-label, randomized three-arm phase III trial. J. Clin. Oncol. 34, 3300-3307 (2016).

46. Gerard, J. P. et al. Clinical complete response (cCR) after neoadjuvant chemoradiotherapy and conservative treatment in rectal cancer. Findings from the ACCORD 12/PRODIGE 2 randomized trial. Radiother. Oncol. 115, 246-252 (2015).

47. Jiao, D. et al. Fluorouracil-based preoperative chemoradiotherapy with or without oxaliplatin for stage II/III rectal cancer: a 3-year follow-up study. Chin. J. Cancer Res. 27, 588-596 (2015)

48. O'Connell, M. J. et al. Capecitabine and oxaliplatin in the preoperative multimodality treatment of rectal cancer: surgical end points from National Surgical Adjuvant Breast and Bowel Project trial R-04. J. Clin. Oncol. 32, 1927-1934 (2014)

49. Schmoll, H. J. et al. Preoperative chemoradiotherapy and postoperative chemotherapy with capecitabine and oxaliplatin versus capecitabine alone in locally advanced rectal cancer: disease-free survival results at interim analysis. J. Clin. Oncol. S32, 3501 (2014).

50. Radojcic, V. et al. Cyclophosphamide resets dendritic cell homeostasis and enhances antitumor immunity through effects that extend beyond regulatory $T$ cell elimination. Cancer Immunol. Immunother. 59, 137-148 (2010).

51. Di Maio, M. et al. Chemotherapy-induced neutropenia and treatment efficacy in advanced non-small-cell lung cancer: a pooled analysis of three randomised trials. Lancet Oncol. 6, 669-677 (2005). 